\begin{tabular}{l|c|l}
\hline \hline Biological and & Vol. 5(2): 281-306, 2021 & $\begin{array}{l}\text { ISSN: 2002-6153 } \\
\text { Biol. Appl. Environ. Res. } \\
\text { www.baerj.com } \\
\text { editor@ } \text { baerj.com }\end{array}$ \\
\hline
\end{tabular}

\title{
Checklist of Fish Hosts of Species of Contracaecum Railliet \& Henry, 1912 (Nematoda: Ascaridida: Anisakidae) in Iraq
}

\author{
Furhan T. Mhaisen $^{1 *}$ \& Kefah N. Abdul-Ameer ${ }^{2}$ \\ ${ }^{1}$ Tegnervägen 6B, Katrineholm 641 36, Sweden \\ ${ }^{2}$ Department of Biology, College of Education for Pure Science, \\ University of Baghdad, Baghdad, Iraq \\ *Corresponding author: mhaisenft@yahoo.co.uk
}

\begin{abstract}
Surveying literature concerning the occurrence of the nematode larval forms of the genus Contracaecum in fishes of Iraq, showed the infection of 44 freshwater and marine fish species in Iraq with such larvae. The infection included larvae of unidentified Contracaecum species, Contracaecum rudolphii type-B and Contracaecum septentrionale Kreis, 1955. The infections were distributed in Tigris, Euphrates and Shatt Al-Arab rivers as well as some of their tributaries, lakes, marshes, drainage networks in addition to many fish ponds and floating cages in different parts of Iraq. This checklist also provided references on some histopathological and biochemical changes, some ecological aspects of the infection, life cycle and scanning electron microscopy. In addition, this checklist includes literature on six species of adult Contracaecum species as well some unidentified species of this genus from 17 bird species from different parts of Iraq, of which both Eurasian bittern Botaurus stellaris and pygmy cormorant Microcarbo pygmaeus were infected with a maximum number of three Contracaecum species as well as unidentified species of this genus.
\end{abstract}

Keywords: Nematoda, Ascaridida, Anisakidae, Contracaecum, Fishes, Iraq

\section{Introduction}

The genus Contracaecum Railliet \& Henry, 1912 has 68 accepted species which belong to the family Anisakidae, order Rhabditida and class Chromodorea of the phylum Nematoda (WoRMS, 2021). Both EOL (2018) and ITIS (2021) listed 25 valid species for this genus. According to GBIF (2021), this genus is considered as a synonym of Hysterothylacium Ward \& Magath, 1917. Members of Contracaecum are significant due to their number of species, wide range of host species involved in their life cycles, and their adverse health impacts on hosts (Shamsi, 2019). Some species have no specificity for fish hosts, so they are able to use a wide variety of fish species as intermediate hosts, and in addition, some nematodes have the ability to survive in "alternative" organisms, known as "paratenic" hosts. These hosts are not required for completion of the life cycle but they can contain infective nematode life stages and be a source of infection (Yanong, 2017). 
Yanong (2017) summarized the general life cycle pattern for nematodes infecting fishes that act as intermediate hosts. Nematode eggs/ larvae (a) pass out in the faces of the definitive host (various species of piscivorous birds and mammals) enter a variety of invertebrate intermediate hosts (b) or a fish intermediate host (c) prior to being eaten by or entering the final host, a fish-eating mammal, bird, or fish (d). Also, Shamsi (2019) gave another summary for the general life cycles of Contracaecum species in which she demonstrated that nematode eggs embryonate into first-stage larvae (L1) within the egg. Then, they develop further and moult to the second larval stage (L2). Eggs or larvae can be ingested by the first intermediate hosts (variety of invertebrates) and then grow in their haemocoel. When the infected invertebrates are eaten by second intermediate hosts (variety of teleost fishes), larvae reach the third larval stage (L3). Various species of piscivorous birds and mammals become infected by predating upon infected fishes and are definitive hosts of Contracaecum. The definitive host may become infected even by consuming the infected first intermediate hosts or the second intermediate hosts (Fig. 1). This general life history pattern is variable and there may be differences in the types of intermediate/definitive hosts among different species of Contracaecum (Shamsi, 2019).

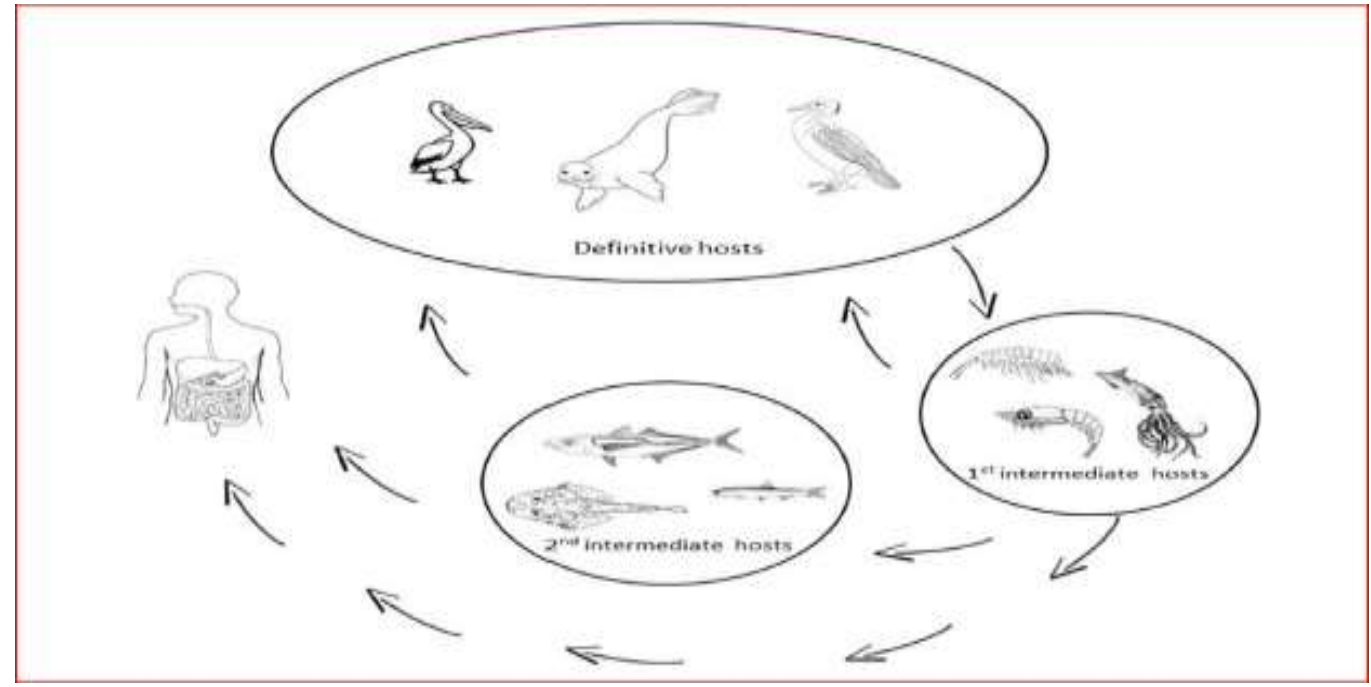

Figure 1: General life cycle of Contracaecum species (from Shamsi, 2019).

The morphological characteristics of the body of the third stage larvae of Contracaecum was demonstrated by Shamsi \& Aghazadeh-Meshgi (2011) and summarized by Shamsi (2019). As the genus' name suggests, these nematodes have two oppositely-directed caecae as part of their digestive system (Figure 2). They also have an excretory pore located at their anterior (cephalic) end. These should be considered the most significant morphological characteristics when differentiating Contracaecum species from the rest of the anisakid nematodes because they are the most consistent at all developmental stages (Shamsi, 2019). In adult Contracaecum 
species, other important features with taxonomic significance include the presence of interlabia and labia, the absence of labial denticulation, rounded eggs with smooth shells, the presence of two spicules, conical tails in both male and females (which are shorter in males) and the presence of post- and pre-cloacal papillae in males (Shamsi, 2019).
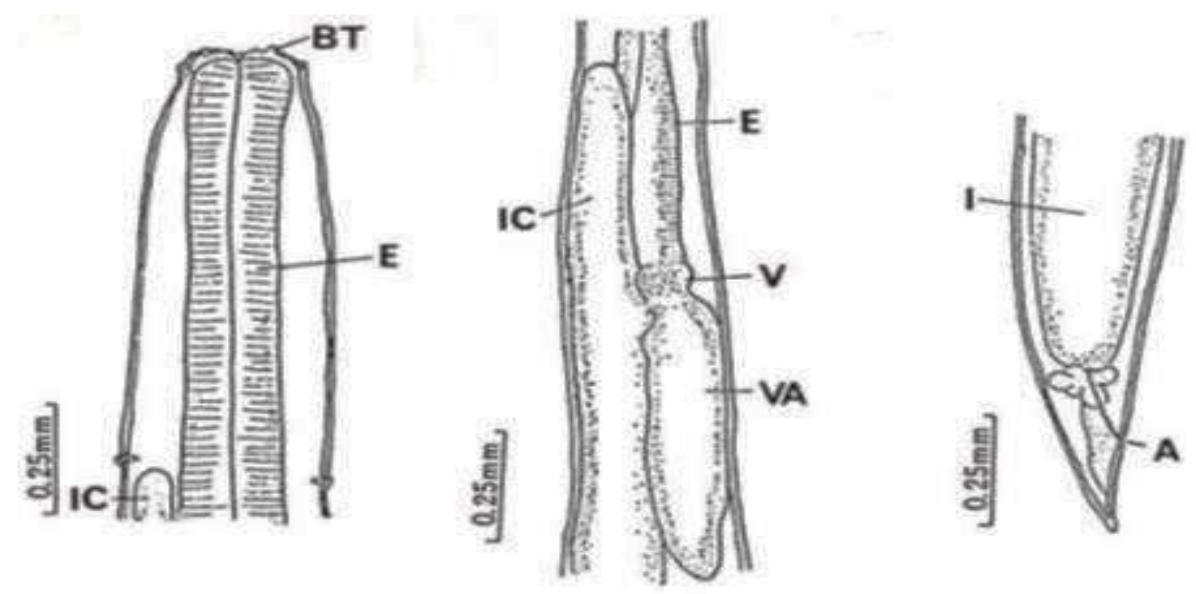

Figure 2: From left to right, anterior, middle and posterior parts of a third-stage larva of Contracaecum sp. A: anus, BT: boring tooth, E: oesophagus, I: intestine, IC: intestinal caecum, V: ventriculus, VA: ventricular appendix (from Nagasawa, 2012).

Contracaecum species are known to be able to have highly pathogenic impacts on both wildlife (fishes, birds, marine mammals) and humans (Shamsi, 2019). If the larvae are accidentally taken by humans by consuming raw or undercooked fish meat, they may cause a zoonotic infection characterized by stomach pains, fever, diarrhoea and vomiting (Girişgin et al., 2012).

In Iraq, Herzog (1969) was the first to detect Contracaecum spp. larvae in nine freshwater fishes from different Iraqi waters and fish markets. After that, many published and unpublished sources were given, most of which dealt with records of different larval forms of Contracaecum species from fishes of Iraq, which will be demonstrated in the results and discussion. However, some articles predicted that these larvae belonged to some adult Contracaecum species (Herzog, 1969; Shamsuddin et al., 1971; Al-Hadithi \& Habish, 1977; Habish, 1977). Such prediction is not acceptable as identification of adult Contracaecum species depends on some features of the reproductive organs, which are no so well developed in the larval forms. However, only recently in Iraq, some molecular investigations were carried out which demonstrated the identification of some adult forms of Contracaecum (Abdullah, 2020; Al-Musaedi, 2020; Abdullah et al., 2021a, b).

In addition to the demonstration of the occurrence of larval forms of Contracaecum in some fishes of Iraq, other researches covered some 
aspects of histopathological changes (Shamsuddin et al., 1971; Habish, 1977; Khalifa et al., 1978; Abdul-Ameer, 1989; Mohamad, 1989; Nawab Al-Deen, 1994; Al-Salim \& Mohamad, 1995; Rahemo \& Nawab Al-Din, 1995; Ali, 2001; AlJadoaa, 2002; Al-Salihi, 2002; Rahemo et al., 2005; Abbas, 2007; Abdul Fraj \& Ftohe, 2008; Al-Darweesh, 2010; Al-Jubouri et al., 2017), biochemical changes (Al-Niaeem, 1999; Ali, 2001; Al-Jadoaa, 2002; Al-Niaeem \& Al-Azizz, 2002; AlSalihi, 2002; Abdul Fraj \& Ftohe, 2008) and some ecological aspects of the infection (Al-Hadithi \& Jawad, 1975; Habish, 1977; Al-Hadithi \& Habash, 1979; Anwar \& Ismail, 1979; Khamees, 1983; Al-Daraji, 1986; Khamees \& Mhaisen, 1988; Mhaisen et al., 1988; Al-Alusi, 1989; Mohamad, 1989; Taher et al., 1994; Al-Awadi, 1997; Al-Nasiri, 2000; Abdullah, 2002; Al-Waaly, 2005; Abdullah \& Mhaisen, 2006; Al-Zubaidy, 2009; Abubakr, 2015; Hussein, 2018; Hathal et al., 2020; Hathal, 2021) as well as life cycles (Habish, 1977) and by using scanning E.M. (Rahemo \& Nawab Al-Din, 2009).

The present article is designed to revise all records on Contracaecum species from fishes of Iraq and provide updated list of their host species in Iraq. It is a continuation of some recent checklists on some groups of fish parasites of Iraq, which included those on Dactylogyrus species (Mhaisen \& Abdul-Ameer, 2019a), ancylodiscoidid and ancyrocephalid species (Mhaisen \& Abdul-Ameer, 2019b), Trichodina species (Mhaisen \& Abdul-Ameer, 2020), Myxobolus species (Mhaisen \& Al-Jawda, 2020) and Lernaea species (Mhaisen \& Abdul-Ameer, 2021).

\section{Sources and Methods}

A total of 120 references (66 published research papers, 35 unpublished M. Sc. theses, one M. Technol. thesis, 14 unpublished Ph. D. theses, three conference abstracts, and one article abstract) dealing with records on Contracaecum species from fishes of Iraq were used to prepare the present article. Data from such references were gathered to provide reliable information on Contracaecum distribution in fishes from different water bodies and fish farms in Iraq as well as fish-Contracaecum list. For fishes, the scientific names were reported as they appeared in their original references but then they were checked with an account on freshwater fishes of Iraq (Coad, 2010). Fish valid scientific names and their authorities were corrected according to a well-known specialized electronic site (Fricke et al., 2021). Valid fish host species are also alphabetically arranged together with their synonyms (if any) and their chronologically arranged references. The nomenclature taxonomy of Contracaecum species was updating according to WoRMS (2021).

\section{Results and Discussion}

\section{Surveys Achieved on Contracaecum Species from Fishes of Iraq}

The available literature concerning the occurrence of Contracaecum species from fishes of Iraq indicated that distribution of such parasites in fishes in different water bodies as well as in fish ponds and farms can be grouped into eight major categories according to localities of collection of the infected fishes. These are: 
1- Tigris River at Nineveh Province (Saleem, 1991; Nawab Al-Deen, 1994; Rahemo \& Nawab Al-Din, 1995; Al-Niaeemi, 1997; Rahemo \& Al-Niaeemi, 2001; Al-Salihi, 2002; Mustafa, 2005; Mustafa et al., 2006; Abdul Fraj \& Ftohe, 2008; Rahemo \& Nawab Al-Din, 2009), Salah Al-Din Province (Abdul-Ameer, 1989; Al-Jawda et al., 2000; Hussein, 2018) and Baghdad Province (Ali et al., 1987b; Balasem et al., 1993; Al-Moussawi, 1997; Hammood, 2017; Abbas, 2019) as well as some tributaries of Tigris River which included Greater Zab River (Ali, 1989; Nawab Al-Deen, 1994; Abdullah, 2002; Abdullah \& Mhaisen, 2006; Shwani, 2009; Shwani \& Abdullah, 2010; Abdullah \& Mhaisen, 2011; Abubakr, 2015), Lesser Zab River (Abdullah, 2002; Abdullah \& Mhaisen, 2006; Shwani \& Abdullah, 2010; Abdullah \& Mhaisen, 2011; Abdullah, 2020; Hathal et al., 2020; Abdullah et al., 2021a, b; Hathal, 2021), Mortuka stream at Erbil Province (Abdullah, 2004), Serchinar stream at Sulaimaniya Province (Rahemo et al., 2005), Sirwan River at Sulaimaniya Province (Abdullah, 2020; Abdullah et al., 2021a, b) and Diyala River (Ali et al., 1987a; Al-Shaikh et al., 1995; Mhaisen et al., 2002).

2- Euphrates River and its branches at Al-Anbar Province (Mhaisen et al., 1997; Al-Sady, 2000; Al-Alusi, 2011; Al-Salmany, 2015), Babylon Province (AlSa'adi, 2007; Al-Zubaidy, 2009; Al-Sa'adi et al., 2012, Mhaisen et al., 2015), Karbala Province (Al-Saadi, 2007; Al-Saadi et al., 2010, 2011), Al-Diwaniah Province (Al-Jadoaa, 2002; Al-Waaly, 2005; Yassin, 2010; Al-Mahi, 2014; AlMahi \& Al-Mayali, 2016, 2017), Thi Qar Province (Rahemo \& Al-Abbadie, 1994; Al-Abbadie, 2006; Al-Kinanny \& Al-Ubaydi, 2017) and Al-Muthanna Province (Al-Asadiy, 2018).

3- Shatt Al-Arab River (Al-Hadithi \& Habish, 1977; Habish, 1977; Al-Hadithi \& Habash, 1979; Anwar \& Ismail, 1979; Al-Hadithi et al., 1980; Mhaisen, 1986; Ali, 2001; Eassa et al., 2014) and some of its branches at Basrah Province which included: Garmat Ali River (Jori, 1998; Abdul-Rahman, 1999; Al-Niaeem, 1999; Al-Niaeem \& Al-Azizz, 2002; Kadhim, 2009; Al-Janae'e, 2010), AlMajidiah River (Mehdi, 1989); Al-Salihiya River (Al-Janae'e, 2010) and Mehaijeran Creek (Khamees, 1983; Mhaisen et al., 1986; Khamees \& Mhaisen, 1988; Mhaisen et al., 1988).

4- Some lakes, depressions and marshes: These included surveys from two lakes in Sulaimaniya province: Darbandikhan Lake (Abdullah, 2005) and Dokan Lake (Abdullah, 1990; Abdullah \& Rasheed, 2004), Al-Tharthar Lake (Khalifa et al., 1978; Al-Saadi, 1986), three lakes in Al-Anbar Province: Al-Rutba Dam Lake (Al-Karboly, 2012), Al-Qadisiya Dam Lake (Al-Alusi, 1989) and AlHabbaniyah Lake (Ali et al., 1988a; Mhaisen et al., 1999), Hemrin Dam Lake in Diyala Province (Balasem et al., 2000), man-made lakes in Salah Al-Din Province (Ali et al., 1988b; Abul-Eis et al., 1989), Al-Tharthar- Tigris Canal in Salah Al-Din Province (Ali \& Shaaban, 1984; Khalifa, 1989), Bahr Al-Najaf depression in Al-Najaf Al-Ashraf Province (Al-Awadi, 1997; Al-Awadi et al., 2010a), Al-Dalmaj Marsh of Al-Diwaniyah Province (Mohammad, 2016), Huwazah Marsh at Maysan Province (Ali, 2008; Al-Musaedi, 2020), Central 
marshes of Thi-Qar Province (Mohammad, 2016) and Al-Hammar Marsh in Basrah Province (Al-Daraji, 1986; Dawood, 1986; Mohamad, 1989; Al-Salim \& Mohamad, 1995; Jori, 2006; Abbas, 2007) as well as in Al-Mashab Marsh in Basrah Province (Al-Tameemi, 2013; Awad \& Al-Tameemi, 2013).

5- Some drainage networks at Babylon Province (Al-Zubaidy, 2009) and AlDiwaniyah Province (Al-Waaly, 2005; Al-Jadoaa, 2008).

6- Fish ponds and farms which included some at Erbil Province (Abdullah, 2004), Baghdad Province (Al-Nasiri, 2000; Al-Nasiri et al., 2002, 2003; Wasit Province (Al-Darweesh, 2010), Babylon Province (Ali et al., 1989; Al-Zubaidy, 1998; AlJadoaa, 2002; Al-Zubaidy, 2009), and Basrah Province (Rasool, 2017) in addition to some floating cages at Babylon Province (Al-Jubouri et al., 2017).

7- Fish markets at Nineveh Province (Al-Mowla, 2010), Erbil Province (Abdullah, 2000), Baghdad Province (Al-Moussawi et al., 2018), fishermen at Al-Najaf AlAshraf Province (Taher et al., 1994), Basrah Province (Al-Hadithi \& Jawad, 1975; Mhaisen, 1986) as well as from fish brought from different areas in mid and southern Iraq: Amara, Habbaniyah, Kut, Tharthar and rivers of Tigris and Euphrates (Shamsuddin et al., 1971).

8- Marine waters of Iraq, which included those from Khor Al-Zubair lagoons (AlDaraji, 1995) and Khor Abdullah (Bannai, 2002).

It is reliable to state here that most records done by Herzog (1969) cannot be categorized to any of the above localities of collection as he mentioned no location for most of the infected fishes in his collections.

The following is a list of the scientific names and the full authority of the infected fishes with Contracaecum species together with their orders and families, based on Fricke et al. (2021).

Class Actinopteri

Order Clupeiformes

Family Clupeidae

Tenualosa ilisha (Hamilton 1822)

Order Cypriniformes

Family Cyprinidae

Arabibarbus grypus (Heckel, 1843)

Barbus lacerta Heckel, 1843

Capoeta damascina (Valenciennes, 1842)

Capoeta trutta (Heckel, 1843)

Carasobarbus luteus (Heckel, 1843)

Carassius auratus (Linnaeus, 1758)

Cyprinion macrostomus Heckel, $1843^{1}$

Cyprinus carpio Linnaeus, 1758

Garra rufa (Heckel, 1843)

Luciobarbus barbulus (Heckel, 1847) 
Luciobarbus esocinus Heckel, 1843

Luciobarbus kersin (Heckel, 1843)

Luciobarbus mystaceus (Pallas, 1814) ${ }^{2}$

Luciobarbus subquincunciatus (Günther, 1868)

Luciobarbus xanthopterus Heckel, 1843

Mesopotamichthys sharpeyi (Günther, 1874)

Family Xenocyprididae ${ }^{3}$

Ctenopharyngodon idella (Valenciennes, 1844)

Family Leuciscidae

Acanthobrama centisquama Heckel, 1843

Acanthobrama marmid Heckel, 1843

Alburnus orontis Sauvage, $1882^{4}$

Alburnus sellal Heckel, 1843

Chondrostoma regium (Heckel, 1843)

Leuciscus vorax (Heckel, 1843)

Squalius cephalus (Linnaeus, 1758)

Squalius lepidus Heckel, 1843

Order Siluriformes

Family Bagridae

Mystus pelusius (Solander, 1794)

Family Siluridae

Silurus glanis Linnaeus, 1758

Silurus triostegus Heckel, 1843

Family Heteropneustidae

Heteropneustes fossilis (Bloch, 1794)

Order Aulopiformes

Family Synodontidae

Saurida undosquamis (Richardson, 1848)

Order Synbranchiformes

Family Mastacembelidae

Mastacembelus mastacembelus (Banks \& Solander, 1794)

Order Pleuronectiformes

Family Soleidae

Brachirus orientalis (Bloch \& Schneider, 1801)

Order Cichliformes

Family Cichlidae

Coptodon zillii (Gervais 1848)

Order Cyprinodontiformes

Family Poeciliidae

Gambusia holbrooki Girard, 1859

Poecilia latipinna (Lesueur, 1821)

Family Aphaniidae

Aphanius stoliczkanus (Day, 1872)

Order Beloniformes 
Family Belonidae Bonaparte 1835

Ablennes hians (Valenciennes, 1846)

Order Mugiliformes

Family Mugilidae

Planiliza abu (Heckel, 1843)

Planiliza subviridis (Valenciennes, 1836)

Order Perciformes

Family Sillaginidae

Sillago sihama (Fabricius, 1775)

Family Sciaenidae

Johnius belangerii (Cuvier 1830)

Otolithes ruber (Bloch \& Schneider 1801)

${ }^{1}$ The specific name of this fish is spelled as macrostomus according to Fricke et al. (2021) but as macrostomum in Froese \& Pauly (2021), WoRMS (2021) as well as in all concerned Iraqi references within this article. The specific name macrostomum was also ascertained through a personal communication with Dr. Jörg Freyhof.

${ }^{2}$ According to Freyhof et al. (2021), Luciobarbus mystaceus has been described by Pallas (1814) from the Kura River in Georgia and this species is a synonym of $L$. capito and some authors refer the species description of B. mystaceus to Heckel (1843), who only identified some barbels from Mesopotamia as B. mystaceus, but did not describe the species.

${ }^{3}$ One reference (Abdul-Rahman, 1999) so far concerning with the occurrence of Contracaecum species in Iraq referred to the grass carp Ctenopharyngodon idella as belonging to the family Cyprinidae. However, Tan \& Armbruster (2018) in their phylogenetic classification, placed this species within the family Xenocyprididae. This is also followed by Fricke et al. (2021) and Froese \& Pauly (2021).

${ }^{4}$ According to Fricke et al. (2021) and Froese \& Pauly (2021), Alburnus orontis is distributed in Turkey and Syria.

5 According to Freyhof et al. (2017), Aphanius dispar in the Arabian Peninsula waters is a complex species and the species in Iraq was Aphanius stoliczkanus (Day, 1872).

\section{Types of Contracaecum Species Known from Fishes of Iraq}

According to Herzog (1969), the first Contracaecum larvae detected from seven fish host species: Arabibarbus grypus (as Barbus grypus), Carasobarbus luteus (as Barbus luteus), Heteropneustes fossilis, Leuciscus vorax (as Aspius vorax), Luciobarbus xanthopterus (as Barbus xanthopterus), Mystus pelusius and Silurus triostegus belonged to $C$. rudolphii Hartwich, 1964, while four fish species: Luciobarbus esocinus (as Barbus esocinus), L. xanthopterus (as B. xanthopterus), Mesopotamichthys sharpeyi (as Barbus sharpeyi) and Planiliza abu (as Mugil abu) 
were infected with unspecified Contracaecum species. Shamsuddin et al. (1971) demonstrated the infection of some freshwater fishes with larvae of Contracaecum species, which they suggested, on basis of their morphology, as belonging to $C$. microcephalum (Rudolphi, 1809), C. multipapillatum (Drasche, 1882) Lucker, 1941 and C. spiculigerum (Rudolphi, 1809). According to WoRMS (2021), C. spiculigerum is considered as a synonym of C. rudolphi. Al-Hadithi \& Habish (1977) and Habish (1977) concluded that larvae of Contracaecum belonged to $C$. microcephalum as they found the adults of this species in the intestine of the purple heron Ardea purpurea. The intestine of this bird also contained L. abu (as Mugil hishni) of which some specimens, from the same collecting area (Shatt Al-Arab River and its branches), were infected with larvae of Contracaecum species.

Recent studies, based on the molecular analysis (Abdullah, 2020, Abdullah et al., 2021a, b) showed that the Contracaecum larval specimens from 10 infected fish species from northern Iraq represented Contracaecum rudolphii type-B. Another recent molecular study (Al-Musaedi, 2020) showed that the isolated Contracaecum sp. from Carasobarbus luteus from Al-Huywazah Marsh, southern Iraq belonged to Contracaecum septentrionale Kreis, 1955.

\section{Contracaecum-Host List}

The following is a list of Contracaecum species so far recorded from fish species of Iraq with their concerned hosts and references.

1- Contracaecum species larvae: Such larvae were reported from 44 valid fish species as in the following alphabetically listed fish species. Ablennes hians: Bannai (2002).

Acanthobrama centisquama: Ali et al. (1987b).

Acanthobrama marmid: Ali et al. (1988b), Abdul-Ameer (1989), Nawab Al-Deen

(1994), Al-Nasiri (2000), Abdullah (2002, 2005), Rahemo \& Nawab Al-Din (2009), Abdullah \& Mhaisen (2011), Al-Salmany (2015).

Alburnus orontis: Al-Sa'adi (2007), Mhaisen et al. (2015).

Alburnus sellal (as Alburnus capito by Al-Nasiri, 2000 and Chalcalburnus sellal by

Abdul-Rahman, 1999): Abdul-Rahman (1999), Al-Nasiri (2000).

Aphanius stoliczkanus (as Aphanius dispar): Al-Awadi (1997), Kadhim, (2009), Al-

Awadi et al. (2010a).

Arabibarbus grypus (as Barbus grypus): Herzog (1969), Shamsuddin et al. (1971),

Al-Hadithi \& Habish (1977), Habish (1977), Ali \& Shaaban (1984), Al-Saadi

(1986), Al-Alusi (1989), Khalifa (1989), Abdullah (1990), Nawab Al-Deen (1994), Al-Awadi (1997), Al-Moussawi (1997), Abdullah (2000), Al-Jawda et al. (2000), Mhaisen et al. (2002), Abdullah \& Rasheed (2004), Al-Saadi (2007), Al-Sa'adi (2007), Al-Awadi et al. (2010a), Al-Saadi et al. (2010), Al-Karboly (2012), Al-Mahi (2014), Mhaisen et al. (2015), Al-Mahi \& Al-Mayali (2017).

Barbus lacerta: Nawab Al-Deen (1994), Al-Alusi (1989).

Brachirus orientalis (as Synaptura orientalis): Bannai (2002). 
Capoeta damascina (as Barbus belayewi): Abdullah (2002), Abdullah \& Mhaisen (2011).

Capoeta trutta (also as Varicorhinus trutta): Abdul-Ameer (1989), Nawab Al-Deen (1994), Rahemo \& Nawab Al-Din (1995), Abubakr (2015).

Carasobarbus luteus (also as Barbus luteus): Herzog (1969), Shamsuddin et al. (1971), Al-Hadithi \& Habish (1977), Habish (1977), Khalifa et al. (1978), Khamees (1983), Al-Daraji (1986), Al-Saadi (1986), Mhaisen (1986), Mhaisen et al. (1986), Ali et al. (1987a), Khamees \& Mhaisen (1988), Ali (1989), Abdullah (1990), Nawab Al-Deen (1994), Abdul-Rahman (1999), Al-Niaeem (1999), Abdullah (2000), Al-Nasiri (2000), Abdullah (2002), Al-Jadoaa (2002), Abdullah \& Rasheed (2004), Al-Waaly (2005), Al-Abbadie (2006), Al-Sa'adi (2007), Abdullah \& Mhaisen (2011), Al-Karboly (2012), Mhaisen et al. (2015), Al-Asadiy (2018).

Carassius auratus: Al-Janae'e (2010).

Chondrostoma regium: Ali et al. (1988a), Abdul-Ameer (1989), Abdullah (1990), Al-Moussawi (1997), Abdullah (2002), Abdullah \& Rasheed (2004), Abdullah (2005), Abdullah \& Mhaisen (2011).

Coptodon zillii (also as Tilapia zillii): Al-Sa'adi (2007), Al-Sa'adi et al. (2012), Mhaisen et al. (2015), Mohammad (2016).

Ctenopharyngodon idella: Abdul-Rahman (1999).

Cyprinion macrostomum: Ali et al. (1987b, 1988b), Abdullah (1990), Abdullah \& Rasheed (2004), Abubakr (2015).

Cyprinus carpio: Shamsuddin et al. (1971), Dawood (1986), Al-Alusi (1989), Abdullah (1990), Al-Zubaidy (1998), Abdul-Rahman (1999), Abdullah (2000), Al-Nasiri (2000), Abdullah (2002), Al-Jadoaa (2002), Al-Nasiri et al. (2002), Abdullah \& Rasheed (2004), Abdullah \& Mhaisen (2006), Al-Darweesh (2010), Yassin (2010), Abdullah \& Mhaisen (2011), Al-Alusi (2011), Eassa et al. (2014), Al-Jubouri et al. (2017), Rasool (2017), Hussein (2018).

Gambusia holbrooki (as Gambusia affinis): Al-Awadi (1997), Al-Awadi et al. (2010a).

Garra rufa: Al-Moussawi (1997), Abdullah (2002), Abdullah \& Mhaisen (2011).

Heteropneustes fossilis (also as Saccobranchus fossilis): Herzog (1969), Al-Hadithi

\& Habish (1977), Habish (1977), Mhaisen (1986), Ali et al. (1987a, b), Mohamad (1989), Balasem et al. (1993), Al-Salim \& Mohamad (1995), AlShaikh et al. (1995), Abdul-Rahman (1999), Ali (2001), Abdullah (2002), Mhaisen et al. (2002), Abdullah (2005), Abdullah \& Mhaisen (2011).

Johnius belangerii: Bannai (2002).

Leuciscus vorax (also as Aspius vorax): Herzog (1969), Al-Hadithi \& Jawad (1975), Al-Hadithi \& Habish (1977), Habish (1977), Khalifa et al. (1978), Khamees (1983), Ali \& Shaaban (1984), Al-Daraji (1986), Al-Saadi (1986), Mhaisen (1986), Mhaisen et al. (1986), Abdul-Ameer (1989), Al-Alusi (1989), Nawab Al-Deen (1994), Al-Moussawi (1997), Mhaisen et al. (1997), AbdulRahman (1999), Al-Jawda et al. (2000), Al-Nasiri (2000), Al-Jadoaa (2002), Al- 
Abbadie (2006), Al-Saadi (2007), Al-Sa'adi (2007), Al-Saadi et al. (2010), AlAlusi (2011), Al-Karboly (2012), Al-Salmany (2015), Mhaisen et al. (2015).

Luciobarbus barbulus (as Barbus barbulus): Abdullah (1990, 2002), Abdullah \& Rasheed (2004), Abdullah \& Mhaisen (2011).

Luciobarbus esocinus (as Barbus esocinus): Herzog (1969) in page 136 but not in 135, Shamsuddin et al. (1971), Khalifa et al. (1978), Abdulllah (1990), Nawab Al-Deen (1994), Abdullah (2000), Balasem et al. (2000), Abdullah \& Rasheed (2004), Abdullah (2005).

Luciobarbus kersin (as Barbus kersin): Abdullah (1990), Abdullah \& Rasheed (2004).

Luciobarbus mystaceus (as Barbus mystaceus): Al-Alusi (1989).

Luciobarbus subquincunciatus (as Barbus subquincunciatus): Abdullah (1990), Abdullah \& Rasheed (2004).

Luciobarbus xanthopterus (as Barbus xanthopterus): Herzog (1969), Shamsuddin et al. (1971), Al-Hadithi \& Habish (1977), Habish (1977), Khalifa et al. (1978), Abdul-Ameer (1989), Al-Alusi (1989), Khalifa (1989), Abdullah (1990), Abdullah (2000), Al-Nasiri (2000), Al-Jadoaa (2002), Abdullah \& Rasheed (2004), Al-Abbadie (2006), Al-Saadi (2007), Al-Sa'adi (2007), Al-Saadi et al. (2010), Al-Alusi (2011), Mhaisen et al. (2015), Hathal et al. (2020), Hathal (2021).

Mastacembelus mastacembelus (also as M. simach): Abdul-Rahman (1999), Abdullah (2002), Al-Salihi (2002), Abdullah (2005), Al-Sa'adi (2007), Ali (2008) as Type BA L3 larva, Al-Mowla (2010), Abdullah \& Mhaisen (2011), Mhaisen et al. (2015).

Mesopotamichthys sharpeyi (also as Barbus sharpeyi): Herzog (1969) in page 136 but not in 135, Shamsuddin et al. (1971), Al-Hadithi \& Habish (1977), Habish (1977), Khalifa et al. (1978), Al-Daraji (1986), Mhaisen (1986), Nawab Al-Deen (1994), Abdul-Rahman (1999), Al-Niaeem \& Al-Azizz (2002), Al-Saadi (2007), Al-Saadi et al. (2010).

Mystus pelusius (also as M. halepensis*): Herzog (1969) in page 136 but not in 135, Al-Hadithi \& Habish (1977), Habish (1977), Ali et al. (1987b)*, Al-Moussawi (1997), Abdul-Rahman (1999), Al-Sa'adi (2007), Mhaisen et al. (2015).

Otolithes ruber (misspelled as Otolithus ruber): Bannai (2002).

Planiliza abu (also as Liza abu, Mugil abu \& Mugil hishni): Herzog (1969) in page 136 but not in 135, Shamsuddin et al. (1971), Al-Hadithi \& Jawad (1975), AlHadithi \& Habish (1977), Habish (1977), Al-Hadithi \& Habash (1979), Anwar \& Ismail (1979), Al-Hadithi et al. (1980), Khamees (1983), Al-Saadi (1986), Mhaisen (1986), Mhaisen et al. (1986), Ali et al. (1987a), Mhaisen et al. (1988), Al-Alusi (1989), Ali et al. (1989), Mehdi (1989), Balasem et al. (1993), Rahemo \& Al-Abbadie (1994), Taher et al. (1994), Al-Awadi (1997), Jori (1998), AbdulRahman (1999), Mhaisen et al. (1999), Al-Nasiri (2000), Al-Sady (2000) p. 36, Al-Jadoaa (2002), Al-Nasiri et al. (2003), Abdullah (2004), Mustafa (2005), AlAbbadie (2006), Mustafa et al. (2006), Al-Saadi (2007), Al-Jadoaa (2008), AlZubaidy (2009), Al-Awadi et al. (2010a), Al-Janae'e (2010), Al-Saadi et al. 
(2010), Yassin (2010), Al-Saadi et al. (2011), Al-Mahi (2014), Al-Mahi \& AlMayali (2016), Al-Kinanny \& Al-Ubaydi (2017), Al-Mahi \& Al-Mayali (2017), Hammood (2017), Hussein (2018), Abbas (2019).

Planiliza subviridis (as Liza subviridis \& Mugil dussumieri): Al-Hadithi \& Habish (1977), Habish (1977), Abdul-Rahman (1999).

Poecilia latipinna: Al-Tameemi (2013), Awad \& Al-Tameemi (2013).

Saurida undosquamis: Bannai (2002).

Sillago sihama: Bannai (2002).

Silurus glanis: Saleem (1991), Al-Niaeemi (1997), Rahemo \& Al-Niaeemi (2001), Al-Salihi (2002), Abdul Fraj \& Ftohe (2008), Al-Mowla (2010) as L3 \& L4 larvae.

Silurus triostegus (also as Parasilurus triostegus): Herzog (1969), Shamsuddin et al. (1971), Al-Hadithi \& Habish (1977), Habish (1977), Khalifa et al. (1978), AlDaraji (1986), Ali et al. (1987a), Abdul-Ameer (1989), Abdul-Rahman (1999), Al-Abbadie (2006), Jori (2006), Abbas (2007), Al-Sa'adi (2007), Ali (2008) as Types BA and BB L3 larvae, Shwani (2009), Al-Janae'e (2010), Shwani \& Abdullah (2010), Mhaisen et al. (2015), Al-Moussawi et al. (2018), Hussein (2018), Hathal et al. (2020), Hathal (2021).

Squalius cephalus (as Leuciscus cephalus): Ali et al. (1987b), Abdullah (2005), Rahemo et al. (2005).

Squalius lepidus (as Leuciscus lepidus): Abdullah (1990, 2002), Abdullah \& Rasheed (2004), Abdullah \& Mhaisen (2011).

Tenualosa ilisha: Al-Janae'e (2010).

Unspecified host: Abul-Eis et al. (1989).

2- Contracaecum rudolphii B larva: This species was recently recognized from the following ten fish species:

Acanthobrama marmid: Abdullah (2020), Abdullah et al. (2021b).

Arabibarbus grypus: Abdullah (2020), Abdullah et al. (2021b).

Capoeta trutta: Abdullah (2020), Abdullah et al. (2021b).

Carasobarbus luteus: Abdullah (2020), Abdullah et al. (2021b).

Chondrostoma regium: Abdullah (2020), Abdullah et al. (2021b).

Cyprinus carpio: Abdullah (2020), Abdullah et al. (2021a).

Luciobarbus barbulus: Abdullah (2020), Abdullah et al. (2021a).

Luciobarbus esocinus: Abdullah (2020), Abdullah et al. (2021a).

Luciobarbus xanthopterus: Abdullah (2020), Abdullah et al. (2021a).

Mastacembelus mastacembelus: Abdullah (2020), Abdullah et al. (2021a).

3- Contracaecum septentrionale Kreis, 1955 larva: This species was recently recognized from Carasobarbus luteus by Al-Musaedi (2020).

It is appropriate to mention here that Ali et al. (2014) indicated that larvae of Contracaecum species in Basrah Province had either a very small caecum, as it the case in those infecting $H$. fossilis (hence known as Contracaecum sp. 2) or a long 
caecum as in the remaining 19 fish host species (hence known as Contracaecum sp. 1). In addition, some adult Contracaecum species were recorded from Khor AlZubair estuary. These included J. belangerii by Al-Daraji (1995) as well as three other fish species (A. hians, S. undosquamis and S. sihama) by Bannai (2002). AlSalim \& Ali (2010) transferred these Contracaecum species to the genus Hysterothylacium Ward \& Magath, 1917 as adult Contracaecum species are known to be parasites of birds and mammals (Fagerholm, 1991).

\section{Adult Contracaecum Species from Birds of Iraq}

In Iraq, some adult Contracaecum species were recorded from some birds in different parts of Iraq such as Baghdad City (Al-Moussawi \& Mohammad, 2011; Al-Moussawi, 2017; Al-Qaisi et al., 2020), Bahr Al-Najaf depression of Al-Najaf Al-Ashraf Province (Al-Awadi et al., 2010b), Al-Sanaf Marsh of Thi-Qar Province (Mohammad, 2014; Mohammad \& Hbaiel, 2019a, b), Al-Hammar Marsh of ThiQar Province (Ali, 2008; Al-Kinanny, 2013), Al-Hammar Marsh of Basrah Province (Ali, 2008), Basrah marshes (Abdullah, 1988; Al-Hadithi \& Abdullah, 1991; Awad et al., 1994; Al-Tameemi, 2013) and Shatt Al-Arab River (Al-Hadithi \& Habish, 1977; Habish, 1977; Abed, 2005; Swadi, 2013; Yaseen, 2018). The bird's valid scientific names followed BirdLife International (2021).

The following is a list of Contracaecum species so far recorded from bird species of Iraq with their concerned hosts and references.

1- Contracaecum microcephalum (Rudolphi, 1809): This species was reported from the following seven bird species:

Ardea purpurea: Al-Hadithi \& Habish (1977), Habish (1977), Awad et al. (1994), Mohammad \& Hbaiel (2019b).

Ardeola ralloides: Ali (2008), Mohammad \& Hbaiel (2019b).

Botaurus stellaris: Ali (2008).

Bubulcus ibis: Swadi (2013).

Egretta grazetta: Awad et al. (1994), Ali (2008), Mohammad \& Hbaiel (2019b).

Microcarbo pygmaeus (reported as Phalocrocorax pygmaeus): Awad et al. (1994).

Pelecanus onocrolalus: Mohammad \& Hbaiel (2019b).

2- Contracaecum micropapillatum (Stossich, 1890): This species was reported from the following three bird species:

Ardea cinerea: Ali (2008).

Botaurus stellaris: Ali (2008).

Nycticorax nycticorax: Mohammad \& Hbaiel (2019a) as free larvae in the proventriculus.

3- Contracaecum multipapillatum (Drasche, 1882) Lucker, 1941: This species was reported from the following three bird species:

Ardeola ralloides: Mohammad \& Hbaiel (2019b).

Egretta grazetta: Mohammad \& Hbaiel (2019b). 
Microcarbo pygmaeus (reported as Phalocrocorax pygmaeus): Awad et al. (1994).

4- Contracaecum ovale (Linstow, 1907): This species was reported from the following three bird species:

Ardea purpurea: Abdullah (1988), Al-Hadithi \& Abdullah (1991), Al-Kinanny (2013).

Botaurus stellaris: Ali (2008).

Egretta gularis: Yaseen (2018).

5- Contracaecum rudolphi Hartwich, 1964 (also reported as C. spiculigerum): This species was reported from the following two bird species:

Microcarbo pygmaeus (reported as Phalocrocorax pygmaeus): Awad et al. (1994), Al-Kinanny (2013).

Phalocrocorax carbo: Al-Moussawi \& Mohammad (2011), Al-Moussawi (2017), Mohammad \& Hbaiel (2019b), Al-Qaisi et al. (2020).

6- Contracaecum septentrionale Kreis, 1955: This species was reported as free larvae from proventriculus of one bird species:

Nycticorax nycticorax: Mohammad \& Hbaiel (2019a).

7- Unidentified adult and fourth larval stages of Contracaecum species were reported from the following 17 bird species:

Ardea alba (reported as Egretta alba): Awad et al. (1994), Al-Awadi et al. (2010b). Ardea cinerea: Ali (2008) as larvae and Al-Tameemi (2013).

Ardea purpurea: Al-Awadi et al. (2010b), Al-Tameemi (2013), Mohammad (2014). Ardeola ralloides: Ali (2008) as larvae, Al-Awadi et al. (2010b), Al-Tameemi (2013), Mohammad (2014).

Botaurus stellaris: Ali (2008) as larvae, Al-Awadi et al. (2010b).

Bubulcus ibis: Al-Tameemi (2013), Mohammad (2014).

Ceryle rudis: Al-Awadi et al. (2010b).

Egretta garzetta: Al-Awadi et al. (2010b), Al-Tameemi (2013), Mohammad (2014), Yaseen (2018).

Egretta gularis: Yaseen (2018).

Himantopus himantopus: Al-Tameemi (2013).

Larus cachinnans: Yaseen (2018).

Larus genei: Al-Tameemi (2013), Mohammad (2014).

Microcarbo pygmaeus (reported as Phalocrocorax pygmaeus): Al-Tameemi (2013).

Nycticorax nycticorax: Al-Tameemi (2013), Mohammad (2014), Yaseen (2018).

Pelecanus onocrotalus: Mohammad (2014).

Phalacrocorax carbo: Abed (2005), Al-Tameemi (2013), Mohammad (2014).

Platalea leucordia: Awad et al. (1994). 


\section{Acknowledgements}

Sincere thanks are due to Dr. Jörg Freyhof of Leibniz Institute for Evolution and Biodiversity Science, Berlin for ascertaining the specific name of the fish Cyprinion macrostomum through a correspondence with Dr. Atheer H. Ali. Sincere thanks are also due to Dr. Atheer H. Ali of the College of Agriculture, University of Basrah, Iraq for forwarding some literature and for his valuable suggestions while revising the manuscript.

\section{References}

Abbas, A.A.-K. (2007). Histopathological studies of some parasites of the Asian catfish, Silurus triostegus (Heckel, 1843) and potassium permanganate on the black molly, Poecilia sphenops (Valenciennes, 1846). Ph. D. Thesis, Coll. Educ., Univ. Basrah: 144 pp. (In Arabic).

Abbas, J.A. (2019). The parasitic fauna of some species of fishes from Tigris River at Al-Autaifia Region, Baghdad Province, Iraq. M. Sc. Thesis, Coll. Educ. Pure Sci. Ibn Al-Haitham, Univ. Baghdad: 140 pp. (In Arabic).

Abdul-Ameer, K.N. (1989). Study of the parasites of freshwater fishes from Tigris River in Salah Al-Dien Province, Iraq. M. Sc. Thesis, Coll. Sci., Univ. Baghdad: 98 pp. (In Arabic).

Abdul Fraj, M.S.A. \& Ftohe, Z.I. (2008). Histological structure of the cyst wall of Contracaecum larva and the histopathology in the stomach of Silurus glanis. Al-Rafidain J. Sci., 19(2): 59-71. (In Arabic). DOI:10.33899/ rjs.2008.41573.

Abdullah, B.H. (1988). A study on parasites of some aquatic birds in Basrah. M. Sc. Thesis, Coll. Educ., Univ. Basrah: 118 pp. (In Arabic).

Abdullah, S.M.A. (1990). Survey of the parasites of fishes of Dokan Lake. M. Sc. Thesis, Coll. Sci., Univ. Salahaddin: 115 pp. (In Arabic).

Abdullah, S.M.A. (2000). Isolation of some parasites in samples of fishes taken from Erbil's market. J. Dohuk Univ., 3(1): 1-6. (In Arabic).

Abdullah, S.M.A. (2002). Ecology, taxonomy and biology of some parasites of fishes from Lesser Zab and Greater Zab rivers in north of Iraq. Ph. D. Thesis, Coll. Educ. (Ibn Al-Haitham), Univ. Baghdad: 153 pp. (In Arabic).

Abdullah, S.M.A. (2004). Comparison between the parasitic infections of fishes caught in two of each of small natural habitats and fish farms in Erbil City. Zanco, 16(4): 43-50. (In Arabic).

Abdullah, S.M.A. (2005). Parasitic fauna of some freshwater fishes from Darbandikhan Lake, north of Iraq. J. Dohuk Univ., 8(1): 29-35.

Abdullah, S.M.A. \& Mhaisen, F.T. (2006). Effect of sex and length of Cyprinus carpio from Lesser Zab River in northern Iraq, and seasonal variations on the infection with some parasites. Rafidain J. Sci., 17(9): 1-9.

Abdullah, S.M.A. \& Mhaisen, F.T. (2011). Infection of fishes from Greater Zab and Lesser Zab rivers in north of Iraq with larvae of nematode Contracaecum spp. Kirkuk Univ. J., Sci. Stud., 6(3): 694-701. (In Arabic). 
Abdullah, S.M.A. \& Rasheed, A.A.M. (2004). Parasitic fauna of some freshwater fishes from Dokan Lake, north of Iraq. II: Endoparasites. Ibn Al-Haitham J. Pure Appl. Sci., 17(5): 1-12.

Abdullah, Y.S. (2020). Ultra-morphological structure and molecular characterization of Contracaecum larvae (Nematode) parasitic of some fishes in Sulaimani Province, Kurdistan Region-Iraq. Ph. D. Thesis, Coll. Sci., Univ. Sulaimani: $130 \mathrm{pp}$.

Abdullah, Y.S.; Abdullah, S.M.A. \& Hussein, R.H. (2021a). Morphology and molecular studies of Contracaecum larvae (Nematoda: Anisakidae) in some fish species from Sulaimani Province, Kurdistan Region, Iraq. Basrah J. Agric. Sci., 34(1): 93-110.

Abdullah, Y.S.; Abdullah, S.M.A. \& Hussein, R.H. (2021b). Ultramorphology and molecular studies of Contracaecum larvae (Nematoda: Anisakidae) collected in five cyprinid fish species from Sulaimani Province, Kurdistan Region-Iraq. Helminthologia, 58(1): 41-58.

Abdul-Rahman, N.M. (1999). Parasites infection in fish from Garmat Ali River and its relation with food items. M. Sc. Thesis, Coll. Agric., Univ. Basrah: 103 pp. (In Arabic).

Abed, J.M. (2005). Biological and ecological survey of some enemies of fishes in three fish farms in Iraq. Ph. D. Thesis, Coll. Agric., Univ. Basrah: 111 pp. (In Arabic).

Abubakr, A.M.-A. (2015). Studies on nematodes of two cyprinid fishes from Greater Zab River, near Aski-Kalak, Kurdistan Region with special reference to the effect of sex, length and season on infection rate. M. Sc. Thesis, Coll. Educ., Univ. Salahaddin: 82 pp.

Abul-Eis, E.S.; Ali, N.M.; Abdul-Ameer, K.N. \& Kadim, L.S. (1989). On the occurrence of fish parasites raised in man-made lakes. II: Helminths. Second Sci. Conf., Fish. Mar. Resour., Basrah: 16-17 May 1989. (Abstract).

Al-Abbadie, F.A.M. (2006). Preliminary study on the parasites of some freshwater fishes in Al-Gharraf River, southern Iraq. J. Thi Qar Univ., 2(3): 17-27. (In Arabic).

Al-Alusi, M.A. (1989). A study of alimentary canal helminths of some species of Iraqi fishes from Al-Qadissiya Dam Lake. M. Sc. Thesis, Coll. Sci., Univ. Baghdad: 110 pp. (In Arabic).

Al-Alusi, M.A. (2011). Survey of some parasitic worms on three fish species from Euphrates River at Al-Haklania District, Al-Anbar Province. Ibn Al-Haitham J. Pure Appl. Sci., 24(1): 69-75. (In Arabic).

Al-Asadiy, Y.D.K. (2018). Detection of the relationship between the intensity of infection Contracaecum sp. (Ascaridoidea) larvae and feeding of freshwater fish caught in Samawahh City. Indian J. Public Health Res. Dev., 9(10): 594599. DOI:10.5958/0976-5506.2018.01197.X. (Abstract only).

Al-Awadi, H.M.H. (1997). Some ecological aspects of the parasitic faunae of fishes and aquatic birds in Bahr Al-Najaf depression, Iraq. Ph. D. Thesis, Coll. Educ. (Ibn Al-Haitham), Univ. Baghdad: 71 pp. 
Al-Awadi, H.M.H.; Mhaisen, F.T. \& Al-Joborae, F.F. (2010a). Parasitic fauna of fishes in Bahr Al-Najaf depression, mid Iraq. Bull. Iraq Nat. Hist. Mus., 11(1): 1-9.

Al-Awadi, H.M.H.; Mhaisen, F.T. \& Al-Joborae, F.F. (2010b). Helminth parasitic fauna of aquatic birds in Bahr Al-Najaf depression, mid Iraq. Bull. Iraq Nat. Hist. Mus., 11(2): 7-15.

Al-Daraji, S.A.M. (1986). Survey of parasites from five species of fishes found in Al-Hammar Marsh. M. Sc. Thesis, Coll. Agric., Univ. Basrah: 130 pp. (In Arabic).

Al-Daraji, S.A.M. (1995). Taxonomical and ecological studies on the metazoan parasites of some marine fishes of Khor Al-Zubair estuary, north-west of the Arabian Gulf. Ph. D. Thesis, Coll. Agric., Univ. Basrah: 182 pp.

Al-Darweesh, A.A.R. (2010). Pathological study of endoparasitic and fungal infections in common carp Cyprinus carpio L. M. Sc. Thesis. Coll. Vet. Med., Univ. Baghdad: 89 pp. (In Arabic).

Al-Hadithi, I.A.W. \& Abdullah, B.H. (1991). Some helminth parasites from three species of aquatic birds in Basrah- Iraq. Basrah J. Agric. Sci., 4(1 \& 2): 261271. (In Arabic).

Al-Hadithi, I.A.W. \& Habash, A.H. (1979). Ecological and biological studies on the larval nematode, Contracaecum sp. a parasite of the fishes in Basrah, Iraq. (2): Seasonal variations. Bull. Coll. Educ., Univ. Basrah, 1: 309-322.

Al-Hadithi, I.A.W. \& Habish, A.H. (1977). Observations on nematode parasite (Contracaecum sp.) in some Iraqi fishes. Bull. Basrah Nat. Hist. Mus., 4: 1725.

Al-Hadithi, I.A. \& Jawad, L.A.J. (1975). Survey on nematode infection of Iraqi fishes. Bull. Basrah Nat. Hist. Mus., 2: 19-25.

Al-Hadithi, I.A.; Habash, A.H. \& Daoud, Y.T. (1980). Seasonal changes of acanthocephalan worms parasitic in mugilid fishes in Shatt Al-Arab. Gulf \& Arab. Penins. Stud., 24: 127-139. (In Arabic).

Ali, A.H. (2001). Pathological effects of helminths parasitic on some local fishes. M. Sc. Thesis, Coll. Agric., Univ. Basrah: 174 pp. (In Arabic).

Ali, A.H. (2008). Taxonomy of helminth parasites in some marine and freshwater fishes and the relation of some of it's with their final hosts in southern of Iraq. Ph. D. Thesis, Coll. Agric., Univ. Basrah: 336 pp. (In Arabic).

Ali, A.H.; Mhaisen, F.T. \& Khamees, N.R. (2014). Checklists of nematodes of freshwater and marine fishes of Basrah Province, Iraq. Mesopot. J. Mar. Sci., 29(2): 71-96.

Ali, B.A.-R. (1989). Studies on parasites of some freshwater fishes from Greater Zab- Iski-Kalak. M. Sc. Thesis, Coll. Sci., Univ. Salahadden: 120 pp. (In Arabic).

Ali, M.D. \& Shaaban, F. (1984). Some species of parasites of freshwater fish raised in ponds and in Tigris- Al-Tharthar Canal Region. Seventh Sci. Conf. Iraqi Vet. Med. Assoc., Mosul: 23-25 Oct. 1984: 44-46. (Abstract). 
Ali, N.M.; Abul-Eis, E.S. \& Abdul-Ameer, K.N. (1988a). Study on the parasites of common carp Cyprinus carpio and other freshwater fishes in Habbaniyah Lake, Iraq. J. Biol. Sci. Res., 19(2): 395-407.

Ali, N.M.; Abul-Eis, E.S. \& Abdul-Ameer, K.N. (1988b). On the occurrence of fish parasites raised in manmade lakes. Sixth Conf. Europ. Ichthyol., Budapest: 15-19 Aug. 1988: 60. (Abstract).

Ali, N.M.; Al-Jafery, A.R. \& Abdul-Ameer, K.N. (1987a). Parasitic fauna of freshwater fishes in Diyala River, Iraq. J. Biol. Sci. Res., 18(1): 163-181.

Ali, N.M.; Salih, N.E. \& Abdul-Ameer, K.N. (1987b). Parasitic fauna of some freshwater fishes from Tigris River, Baghdad, Iraq. IV: Nematoda. J. Biol. Sci. Res., 18(3): 35-45.

Ali, N.M.; Mhaisen, F.T.; Abul-Eis, E.S. \& Kadim, L.S. (1989). Helminth parasites of the mugilid fish Liza abu (Heckel) inhabiting Babylon Fish Farm, Hilla, Iraq. Proc. $5^{\text {th }}$ Sci. Conf., Sci. Res. Counc., 5(2): 225-233.

Al-Jadoaa, N.A.A. (2002). The parasitic infections and pathological changes of some local and cultured fishes from Al-Qadisiya and Babylon provinces. Ph. D. Thesis, Coll. Educ., Al-Qadisiya Univ.: 158 pp. (In Arabic).

Al-Jadoaa, N.A. (2008). Study of the parasitic infection of Liza abu fish in drainage water at Al-Diwaniya Province. J. Babylon Univ., 15(1): 256-263. (In Arabic).

Al-Janae'e, A.M.S. (2010). Parasites of some Iraqi fishes in two localities varied in their trophic levels in inland water of Basrah. M. Sc. Thesis, Coll. Agric., Univ. Basrah: 227 pp. (In Arabic).

Al-Jawda, J.M.; Balasem, A.N.; Mhaisen, F.T. \& Al-Khateeb, G.H. (2000). Parasitic fauna of fishes from Tigris River at Salah Al-Deen Province, Iraq. Iraqi J. Biol. Sci., 19 \& 20: 16-24.

Al-Jubouri, M.O.A.; Al-Niaeem, K.S. \& Taher, M.M. (2017). Clinical diagnosis of some diseases and parasites infected common carp (Cyprinus carpio) cultivated in floating cages at Babylon Province. Euphrates J. Agric. Sci. (Second Vet. Conf.): 229-244.

Al-Karboly, R.W.K. (2012). The dispersal on intestinal parasites in fish in Al-Rutba Dam. Iraqi J. Desert Stud., 4(1): 1-4. (In Arabic).

Al-Kinanny, Z.A.H. (2013). Parasitic helminthes in some aquatic birds in AlHammar Marsh of Thi-Qar governorate with reference to some of environmental aspects of the parasites birds Bubulcus ibis. M. Sc. Thesis, Coll. Educ. Pure Sci., Univ. Thi-Qar: 106 pp. (In Arabic).

Al-Kinanny, Z.A.H. \& Al-Ubaydi, N.A.H. (2017). Seasonal changes and their impact on the incidence internal parasites of fish Liza $a b u$ in the-Qar governorate. J. Al-Qadisiyah Pure Sci., 22(1): 41-47. (In Arabic).

Al-Mahi, A.M.Y. (2014). The usage of bio-accumulation of heavy metals in tissues of parasitic helminthes of two types of fresh waters fishes in Al-Qadisiya governorate. Ph. D. Thesis, Coll. Educ., Al-Qadisiya Univ.: 132 pp. (In Arabic). 
Al-Mahi, A.M.Y. \& Al-Mayali, H.M. (2016). Identification of some intestinal helminthes in Barbus grypus, Liza abu and study of histopathological changes result from infection. J. Al-Qadisiyah Pure Sci., 21(1): 96-115. (In Arabic).

Al-Mahi, A.M.Y. \& Al-Mayali, H.M. (2017). Measurements of some heavy metals in tissues infected by intestinal worms of two kind of fishes in Al-Qadisiyah Province. Al-Qadisiyah J. Vet. Med. Sci., 16(3): 115-124. 6th Conf. ( $1^{\text {st }}$ Int.) 27-28 Sep. 2017. DOI:10.29079/vol17iss 1art486.

Al-Moussawi, A.A. (1997). A taxonomic comparison between some species of family Cyprinidae and family Bagridae and their infection with endoparasites. M. Sc. Thesis, Coll. Sci., Univ. Baghdad: 86 pp. (In Arabic).

Al-Moussawi, A.A. (2017). Insights at morphological features of Contracaecum rudolphii Hartwich, 1964 (Nematoda: Anisakidae) as revealed by scanning electron microscope (SEM). J. Entomol. Zool. Stud., 5(3): 116-119.

Al-Moussawi, A.A. \& Mohammad, M.K. (2011). Contracaecum rudolphii Hartwich, 1964 (Nematoda: Anisakidae) in the cormorant Phalacrocorax carbo (Linnaeus, 1758) wintering in Baghdad area: A new host record in Iraq. Bull. Iraq Nat. Hist. Mus., 11(3): 7-16.

Al-Moussawi, A.A.; Hadi, A.M. \& Macawi, Z.A. (2018). Diagnosis of some parasites of Asian catfish Silurus triostegus (Heckel, 1843). Adv. Biores., 9(3): 86-90.

Al-Mowla, S.A.T. (2010). Biochemical parameters in some nematodes, which infect some vertebrates. Ph. D. Thesis, Coll. Sci., Univ. Mosul: 229 pp. (In Arabic).

Al-Musaedi, A.I.C. (2020). Morphological and molecular study of gastrointestinal helminthes parasites of some fish species in Al-Huwayzah Marsh, Misan/ Iraq. M. Sc. Thesis, Coll. Sci., Univ. Misan: 97 pp.

Al-Nasiri, F.S. (2000). Parasitic infections of fishes in a man-made lake at AlAmiriya Region, Baghdad. M. Sc. Thesis, Coll. Educ. (Ibn Al-Haitham), Univ. Baghdad: 133 pp. (In Arabic).

Al-Nasiri, F.S.; Mhaisen, F.T. \& Al-Nasiri, S.K. (2002). Parasitic infections of the common carp, Cyprinus carpio in a man-made lake at Baghdad Region. Iraqi J. Agric. (Spec. Issue), 7(1): 175-181.

Al-Nasiri, F.S.; Mhaisen, F.T. \& Al-Nasiri, S.K. (2003). Parasites of the grey mullet Liza abu in a man-made lake at Baghdad Region. Iraqi J. Agric. (Spec. Issue), 8(1): 133-140.

Al-Niaeem, K.S.K. (1999). Histopathological and biochemical effects of some helminths parasitic in three Iraqi freshwater fishes. M. Sc. Thesis, Coll. Agric., Univ. Basrah: 73 pp. (In Arabic).

Al-Niaeem, K.S. \& Al-Azizz, S.A.A. (2002). The influence of parasitism in Barbus sharpeyi on protein and lipid content. Basrah J. Vet. Res., 1(1): 27-32. (In Arabic).

Al-Niaeemi, B.H.S. (1997). A study on parasites of the fish Silurus glanis L., from Tigris River in Mosul City with special reference to the histopathological 
effects caused by some infections. M. Sc. Thesis, Coll. Sci., Univ. Mosul: 116 pp. (In Arabic).

Al-Qaisi, A.Q.; Al-Warid, H.S. \& Al-Moussawi, A.A. (2020). Molecular characterization of Contracaecum rudolphii Hartwich, 1964 (Nematoda: Anisakidae) from the cormorant Phalacrocorax carbo in Iraq. Bull. Iraq Nat. Hist. Mus., 16(2): 135-150. DOI:10.26842/binhm.7.2020.16.2.0135.

Al-Saadi, A.A.J.J. (1986). A survey of alimentary canal helminths of some species of fishes from Tharthar Lake. M. Sc. Thesis, Coll. Sci., Univ. Baghdad: 94 pp. (In Arabic).

Al-Saadi, A.A.J.J. (2007). Ecology and taxonomy of parasites of some fishes and biology of Liza abu from Al-Husainia Creek in Karbala Province, Iraq. Ph. D. Thesis, Coll. Educ. (Ibn Al-Haitham), Univ. Baghdad: 155 pp. (In Arabic).

Al-Saadi, A.A.J.J.; Mhaisen, F.T. \& Hasan, H.R. (2010). Endoparasites of seven fish species from Al-Husainia Creek, Karbala Province, mid Iraq. Basrah J. Agric. Sci., 23 (Spec. Issue 2): 139-146.

Al-Saadi, A.A.J.; Mhaisen, F.T. \& Hasan, H.R. (2011). Some aspects of the reproductive biology of the mugilid fish Liza abu (Heckel) in Al-Husainia Creek, Karbala Province, Iraq and effect of parasitism on its gonadosomatic index. Iraqi J. Agric. Res. (Spec. Issue), 16(3): 166-173. (In Arabic).

Al-Sa'adi, B.A.-H.E. (2007). The parasitic fauna of fishes of Euphrates River: Applied study in Al-Musaib City. M. Technol. Thesis, Al-Musaib Technic. Coll., Found. Technic. Educ.: 102 pp. (In Arabic).

Al-Sa'adi, B.A.; Mhaisen, F.T. \& Al-Rubaie, A.-R.L. (2012). The first parasitological report on the redbelly tilapia Tilapia zillii (Gervais, 1848) in Iraq. Proc. $1^{\text {st }}$ Sci. Symp. Iraq Nat. Hist. Mus. Baghdad: 20 June 2012: 1-6.

Al-Sady, R.S. (2000). Description of a new species of Acanthocephala (Neoechinorhynchus iraqensis) and some ecological aspects of its infection to the mugilid fish Liza abu from Al-Faluja Region, Al-Anbar Province with observations on the experimental infection. M. Sc. Thesis, Coll. Educ. (Ibn Al-Haitham), Univ. Baghdad: 84 pp. (In Arabic).

Al-Salihi, M.S.A.-F.M. (2002). Histopathological and histochemical studies on the cysts of helminth larvae in some vertebrates in Nenavah governorate. Ph. D. Thesis, Coll. Sci., Univ. Mosul: 145 pp. (In Arabic).

Al-Salim, N.K. \& Ali, A.H. (2010). Description of eight nematode species of the genus Hysterothylacium Ward et Magath, 1917 parasitized in some Iraqi marine fishes. Basrah J. Agric. Sci., 23 (Spec. Issue 2): 115-137.

Al-Salim, N.K. \& Mohamad, E.T. (1995). Studies on some parasites of the stinging catfish (Heteropneustes fossilis) from Al-Hammar Marsh- Basrah. A) Infection of Heteropneustes fossilis by four parasites and their effects on the fish infected. Mar. Mesopot., 10(1): 155-170. (In Arabic).

Al-Salmany, S.O.K. (2015). Parasitic infections of some fish species from Euphrates River at Al-Qaim City, Anbar Province. M. Sc. Thesis, Coll. Sci., Univ. Tikrit: 193 pp. (In Arabic). 
Al-Shaikh, S.M.; Mhaisen, F.T.; Al-Khateeb, G.H.; Balasem, A.N. \& Mansoor, N.T. (1995). Collection of some fish parasites from the lower reaches of Diyala River, mid Iraq. J. Environ. Sci. Health, A 30(8): 1707-1715.

Al-Tameemi, I.A.A. (2013). Helminthes parasitized on some aquatic birds \& the importance of insects in the life cycle of some of them in Basrah Province. Ph. D. Thesis, Coll. Educ. Pure Sci., Univ. Basrah: 222 pp. (In Arabic).

Al-Waaly, A.B.M. (2005). A comparative study for parasites of Barbus luteus fishes in Al-Daghara River and drainage water. M. Sc. Thesis, Coll. Educ., Univ. Al-Qadisyia: 101 pp. (In Arabic).

Al-Zubaidy, A.B. (1998). Studies on the parasitic fauna of carps in Al-Furat Fish Farm, Babylon Province, Iraq. Ph. D. Thesis, Coll. Sci., Univ. Babylon: 141 pp. (In Arabic).

Al-Zubaidy, A.B. (2009). Prevalence and densities of Contracaecum sp. larvae in Liza abu (Heckel, 1843) from different Iraqi water bodies. J. King Abdulaziz Univ., Mar. Sci., 20: 3-17.

Anwar, S.M. \& Ismail, A.W. (1979). Dispersion pattern of parasite population of mugil fish. Pak. J. Zool., 11(12): 211-214.

Awad, A.H. \& Al-Tameemi, I.A.A. (2013). The role of local fishes as an intermediate or paratenic hosts for some parasites of aquatic birds in Basrah Province. Basrah J. Agric. Sci., 26 (Spec. Issue 2): 205-210. (In Arabic).

Awad, A.H.; Abdullah, B.H. \& Al-Mayah, S.H. (1994). Some nematodes parasitized in seven species of aquatic birds in Basrah, Iraq. Basrah J. Sci., B, 12(1): 63-70.

Balasem, A.N.; Mohammad-Ali, N.R.; Adday, T.K.; Ali, A.K. \& Waheed, I.K. (2000). Parasitological survey on fish in Hemrin Dam Lake, Province of Diyala. J. Diyala, 1(8 Part 1): 104-114. (In Arabic).

Balasem, A.N.; Mhaisen, F.T.; Al-Shaikh, S.M.J.; Al-Khateeb, G.H.; Asmar, K.R. \& Adday, T.K. (1993). Survey of fish parasites from Tigris River at AlZaafaraniya, south of Baghdad, Iraq. Mar. Mesopot., 8(3): 226-235.

Bannai, M.A.-A. (2002). Parasites of some marine fishes of Khor Abdulla, northwest Arabian Gulf. M. Sc. Thesis, Coll. Educ., Univ. Basrah: 103 pp. (In Arabic).

BirdLife International (2021). Available from: http://www.birdlife.org/ (accessed 9 April 2021).

Coad, B.W. (2010). Freshwater fishes of Iraq. Pensoft Publ., Sofia: 274 pp. + 16 pls. www.briancoad.com.

Dawood, I.H. (1986). The biology of common carp (Cyprinus carpio L.) in Hor AlHammar, southern Iraq. M. Sc. Thesis, Coll. Agric., Univ. Basrah: 94 pp. (In Arabic).

Eassa, A.M.; Al-Jenaei, A.M.; Abdul-Nabi, Z.A.; Abood, M.A.; Kzaal, R.S. \& Aliwy, Y.J. (2014). Comparative ecological study of pathogens structure between wild and cultured common carp Cyprinus carpio L. in Basrah. Marsh Bull., 9(2): 107-123. 
EOL (2018). Encyclopedia of Life on-line database, http//www.eol.org. (Accessed 15 July 2018).

Fagerholm, H.P. (1991). Systematic implications of male caudal morphology in ascaridoid nematode parasites. Syst. Parasitol., 19: 215-228.

Freyhof, J.; Kaya, C. \& Ali, A. (2021). A critical checklist of the inland fishes native to the Euphrates and Tigris drainages. In: Jawad, L.A. (ed.). Tigris and Euphrates rivers: Their environment from headwaters to mouth. Aquatic Ecology Series II, Chapter 35: 40 pp. In Press.

Freyhof, J.; Weissenbacher, A. \& Geiger, M. (2017). Aphanius kruppi, a new killifish from Oman with comments on the A. dispar species group (Cyprinodontiformes: Aphaniidae). Zootaxa, 4338(3): 557-573. DOI:10.11646/zootaxa.4338.3.10.

Fricke, R.; Eschmeyer, W.N. \& Van der Laan, R. (eds.) (2021). Eschmeyer's Catalog of Fishes: Genera, Species, References. http://researcharchive.calacademy.org/ research/ichthyology/catalog/fishcatmain.asp. (Updated 13 April 2021).

Froese, R. \& Pauly, D. (eds.) (2021). FishBase. World Wide Web electronic publication. www.fishbase.org, version (02/2021).

GBIF (2021). Global Biodiversity Information Facility, on-line database, http://www.gbif.org. (Accessed 9 April 2021).

Girişgin, A.O.; Alasonyalilar-Demirer, A. \& Girişgin, O. (2012). A case of Contracaecum sp. (Ascaridida: Anisakidae) infection in Dalmatian pelican (Pelecanus crispus). Kafkas Univ. Vet. Fak. Derg., 18(Suppl. A): 227-229.

Habish, A.H. (1977). Ecological and biological studies on the larval nematode, Contracaecum sp. a parasite of the fishes in Basrah, Iraq. M. Sc. Thesis, Coll. Sci., Univ. Basrah: 98 pp.

Hammood, N.W. (2017). Investigation of some parasitic and bacterial infections in some fish species of Tigris River at Baghdad City. M. Sc. Thesis, Coll. Sci., Univ. Tikrit: 141 pp. (In Arabic).

Hathal, B.A. (2021). Survey study and effect of different parasite species on growth of two fish host species from Lesser Zab River. M. Sc. Thesis, Coll. Agric., Univ. Kirkuk: 77 pp.

Hathal, B.A.; Nawwab Al-Deen, F.M. \& Bilal, S.J. (2020). Helminth parasite fauna of two freshwater fishes and relation to their sex and length from Lesser Zab River Northern Iraq. Euphrates J. Agric. Sci., 12(2): 179-193.

Herzog, P.H. (1969). Untersuchungen über die parasiten der süßwasserfische des Irak. Arch. Fischereiwiss., 20(2/3): 132-147.

Hussein, M.A. (2018). Parasitic in infection of three fish species passing through Tikrit City/ Salah Al-Din Province. M. Sc. Thesis, Coll. Educ. Women, Univ. Tikrit: 89 pp. (In Arabic).

ITIS (2021). Integrated Taxonomic Information System on-line database, http://www.itis.gov. (Accessed 9 April 2021). 
Jori, M.M. (1998). Study of the parasites of two mugilid fish species and the effect of some on the blood parameters. M. Sc. Thesis, Coll. Agric., Univ. Basrah: 136 pp. (In Arabic).

Jori, M.M. (2006). Parasitic study on the Asian catfish Silurus triostegus (Heckel, 1843) from Al-Hammar marshes, Basrah, Iraq. Ph. D. Thesis, Coll. Educ., Univ. Basrah: 192 pp.

Kadhim, A.H. (2009). Disease agents of four fish species in Basrah Province. M. Sc. Thesis, Coll. Agric., Univ. Basrah: 102 pp. (In Arabic).

Khalifa, K.A. (1989). Incidence of parasitic infestation of fishes in Iraq. Pak. Vet. J., 9(2): 66-69.

Khalifa, K.A.; Hassan, F.K.; Atiah, H.H. \& Latif, B.M.A. (1978). Parasitic infestation of fishes in Iraqi waters. Iraqi J. Biol. Sci., 6(1): 58-63.

Khamees, N.R. (1983). A study of the parasites of Carasobarbus luteus (Heckel), Liza abu (Heckel) and Aspius vorax Heckel from Mehaijeran canal, south of Basrah. M. Sc. Thesis, Coll. Agric., Univ. Basrah: 148 pp. (In Arabic).

Khamees, N.R. \& Mhaisen, F.T. (1988). Ecology of parasites of the cyprinid fish Carasobarbus luteus from Mehaijeran Creek, Basrah. J. Biol. Sci. Res., 19(2): 409-419.

Mehdi, D.S. (1989). Effect of parasites on the biochemical constituents of fishes Liza abu. M. Sc. Thesis, Coll. Educ., Univ. Basrah: 98 pp. (In Arabic).

Mhaisen, F.T. (1986). Records of some fish parasites from Shatt-Al-Arab River and the north west of the Arab Gulf. Bull. Basrah Nat. Hist. Mus., 6(1): 111-124.

Mhaisen, F.T. \& Abdul-Ameer, K.N. (2019a). Checklists of Dactylogyrus species (Monogenea) from fishes of Iraq. Biol. Appl. Environ. Res., 3(1): 1-36.

Mhaisen, F.T. \& Abdul-Ameer, K.N. (2019b). Checklists of species of ancylodiscoidid and ancyrocephalid monogeneans from fishes of Iraq. Basrah J. Agric. Sci., 32(Spec. 2): 45-62.

Mhaisen, F.T. \& Abdul-Ameer, K.N. (2020). Checklists of the species of Trichodina Ehrenberg, 1830 (Ciliophora: Oligohymenophorea: Peritrichida) from fishes of Iraq. Biol. Appl. Environ. Res., 4(2): 196-228.

Mhaisen, F.T. \& Abdul-Ameer, K.N. (2021). Checklist of fish hosts of species of Lernaea Linnaeus, 1758 (Hexanauplia: Cyclopoida: Lernaeidae) in Iraq. Biol. Appl. Environ. Res., 5(1): 53-73. DOI:10.51304/baer.2021.5.1.53.

Mhaisen, F.T. \& Al-Jawda, J.M. (2020). Checklists of the species of Myxobolus Bütschli, 1882 (Cnidaria: Myxozoa: Myxobolidae) from fishes of Iraq. Biol. Appl. Environ. Res., 4(2): 127-166.

Mhaisen, F.T.; Al-Rubaie, A.L. \& Al-Sa'adi, B.A. (2015). Nematodes and acanthocephalans of fishes from the Euphrates River at Al-Musaib City, Babylon Province, mid Iraq. Am. J. Biol. Life Sci., 3 (4): 108-115.

Mhaisen, F.T.; Al-Saadi, A.A.J. \& Al-Shamma'a, A.A. (1999). Some observations on fish parasites of Habbaniya Lake. Ibn Al-Haitham J. Pure Appl. Sci., 12(1): 62-67. 
Mhaisen, F.T.; Al-Salim, N.K. \& Khamees, N.R. (1986). The parasitic fauna of two cyprinids and a mugilid fish from Mehaijeran Creek, Basrah. J. Biol. Sci. Res., 17(3): 63-73.

Mhaisen, F.T.; Al-Salim, N.K. \& Khamees, N.R. (1988). Occurrence of parasites of the freshwater mugilid fish Liza abu (Heckel) from Basrah, southern Iraq. J. Fish Biol., 32(4): 525-532.

Mhaisen, F.T.; Al-Khateeb, G.H.; Balasem, A.N. \& Mutar, A.J. (1997). On a collection of some fish parasites from Euphrates River, Anbar Province, Iraq. Babylon Univ. J., Pure Appl. Sci., 2(3): 267-272.

Mhaisen, F.T.; Balasem, A.N.; Al-Khateeb, G.H.; Asmar, K.R. \& Adday, T.K. (2002). A second collection of fish parasites and fungi from the lower reaches of Diyala River, mid Iraq. Vet., 12(1): 24-32.

Mohamad, E.T. (1989). Study on parasites of the stinging catfish Heteropneustes fossilis (Bloch, 1797) from Al-Hammar Marsh, Basrah. M. Sc. Thesis, Coll. Agric., Univ. Basrah: 103 pp. (In Arabic).

Mohammad, M.K. (2016). The parasitic fauna of the exotic fish Tilapia zillii in the Middle and South of Iraq. Int. J. Curr. Microbiol. Appl. Sci., 5(3): 93-96.

Mohammad, Z.A.A. (2014). Ectoparasites and helminthes of some aquatic birds in Al-Sanaf Marsh, southern Thi-Qar Province/Iraq. Ph. D. Thesis, Coll. Educ. Pure Sci., Uni. Basrah: 218 pp.

Mohammad, Z. A.-A. \& Hbaiel, M.K. (2019a). Morphological and Molecular study of Contracaecum larvae with a new record of Contracaecum Septentrionale in Al-Sanaf Marsh southern Thi-Qar Province, Iraq. Indian J. Public Health Res. Develop., 10(10): 1271-1276.

Mohammad, Z.A.-A. \& Hbaiel, M.K. (2019b). Isolate and identify of Contracaecum spp. nematodes from some aquatic bird species by using light microscope and scanning electron microscope. J. Educ. Pure Sci., Univ. ThiQar, 9(2): 180-192. DOI:10.32792/utq.jceps.09.02.19.

Mustafa, Y.A.S. (2005). Intestinal parasites of mugilid fish Liza abu in Mosul and study of experimental infection with Goussia carpelli in common carp Cyprinus carpio and the effect of methionine on the infection. M. Sc. Thesis, Coll. Vet. Med., Univ. Mosul: 83 pp. (In Arabic).

Mustafa, Y.A.S.; Al-Kenanny, E.R.O. \& Salih, N.E. (2006). A survey study on the intestinal parasites of Liza abu in Mosul. Proc. $4^{\text {th }}$ Sci. Conf., Coll. Vet. Med., Univ. Mosul: 229-241. (In Arabic).

Nagasawa, K. (2012). The biology of Contracaecum osculatum sensu lato and $C$. osculatum A (Nematoda: Anisakidae) in Japanese waters: A review. Biosphere Sci., 51: 61-69.

Nawab Al-Deen, F.M. (1994). Studies on the nematode parasites in many species of freshwater fishes in Iraq. M. Sc. Thesis, Coll. Sci., Univ. Mosul: 116 pp. (In Arabic).

Rahemo, Z.I.F. \& Al-Abbadie, F.A.M. (1994). Parasites of Liza abu (Heckel) from Al-Gharaf River, Thi-Qar Region- Iraq. J. Educ. Sci., 18: 48-59. (In Arabic). 
Rahemo, Z.I.F. \& Al-Niaeemi, B.H.S. (2001). Parasites of Silurus glanis inhabiting Tigris River passing Mosul City. J. Al-Qadisiya, Pure Sci., 6(3): 116-125. (In Arabic).

Rahemo, Z.I.F. \& Nawab Al-Din, F.M. (1995). The histopathology caused by two nematode larvae in two species of fishes. Türk. Parazitol. Derg., 19(4): 571575.

Rahemo, Z.I.F. \& Nawab Al-Din, F. (2009). Morphological observation at SEM on three species of nematodes from Iraqi fishes. J. Kirkuk Univ., Sci. Stud., Spec. Issue 4(3): 13-21.

Rahemo, Z.I.F.; Hamdi, B.A.; Al-Khateeb, I.A.M. \& Aziz, F.M. (2005). Histological changes in the liver of the freshwater fish, Leuciscus cephalus caught from Serchinar stream, infected with Contracaecum larvae. Riv. Parassitol., 22(66), No. 3: 185-190.

Rasool, A.H. (2017). Detection of some parasites and study their effects on certain blood and biochemical parameters of Cyprinus carpio in south of Iraq. M. Sc. Thesis, Coll. Vet. Med, Univ. Basrah: 102 pp.

Saleem, K.M. (1991). A study on some nematodes of the freshwater fishes of Tigris River in Mosul and Lake of Mosul Dam. Mesopot. J. Agric., 23(3): 5-8. (In Arabic).

Shamsi, S. (2019). Parasite loss or parasite gain? Story of Contracaecum nematodes in antipodean waters. Parasite Epidemiol. Control, 3 e00087. DOI:10.1016/ j.parepi.2019.e00087.

Shamsi. S. \& Aghazadeh-Meshgi, M. (2011). Morphological and genetic characterisation of selected Contracaecum (Nematoda: Anisakidae) larvae in Iran. Iran. J. Fish. Sci., 10(2): 356-361.

Shamsuddin, M.; Nader, I.A. \& Al-Azzawi, M.J. (1971). Parasites of common fishes from Iraq with special reference to larval form of Contracaecum (Nematoda: Heterocheilidae). Bull. Biol. Res. Cent., Baghdad, 5: 66-78.

Shwani, A.A.A. (2009). The parasitic fauna of Asian catfish Silurus triostegus (Heckel, 1843) from Greater Zab River- Kurdistan Region- Iraq. M. Sc. Thesis, Coll. Sci. Educ., Univ. Salahaddin: 75 pp.

Shwani, A.A.A. \& Abdullah, S.M.A. (2010). Endoparasites of the Asian catfish Silurus triostegus (Heckel, 1843) from Greater Zab River- Kurdistan RegionIraq. J. Duhok Univ., 13(1): 172-179.

Swadi, B.F. (2013). Helminthes parasitized on some aquatic birds which occupied on Shatt Al-Arab coast north of Basrah governorate. M. Sc. Thesis, Coll. Educ. Pure Sci., Univ. Basrah: 110 pp. (In Arabic).

Taher, J.H.; Jabouri, S.S. \& Abid, N.H. (1994). Epidemiology of Contracaecum larvae to Liza abu (Heckel) in Al-Najaf Region/ Iraq. Tech. Res. J., 7(19): 277-290. (In Arabic).

Tan, M. \& Armbruster, J.W. (2018). Phylogenetic classification of extant genera of fishes of the order Cypriniformes (Teleostei: Ostariophysi). Zootaxa, 4476(1): 006-039. DOI:10.11646/zootaxa.4476.1.4. 
WoRMS (2021). World Register of Marine Species at http://www. marinespecies.org at VLIZ. (Accessed 9 April 2021).

Yanong, R.P.E. (2017). Nematode (roundworm) infections in fish. Univ. Florida/ Inst. Food Agric. Sci. Ext., Circ. 91: 9 pp.

Yaseen, A.M. (2018). Helminthes parasitizing on some aquatic birds in the Faw Town south eastern of Basrah governorate, Iraq. M. Sc. Thesis, Coll. Educ. Pure Sci., Univ. Basrah: 115 pp. (In Arabic).

Yassin, A.M. (2010). Isolation and identification of the parasites of Liza abu and Cyprinus carpio in Al-Shenafya River. J. Wasit Univ. Sci. Med., 3(1): 34-43. (In Arabic). 